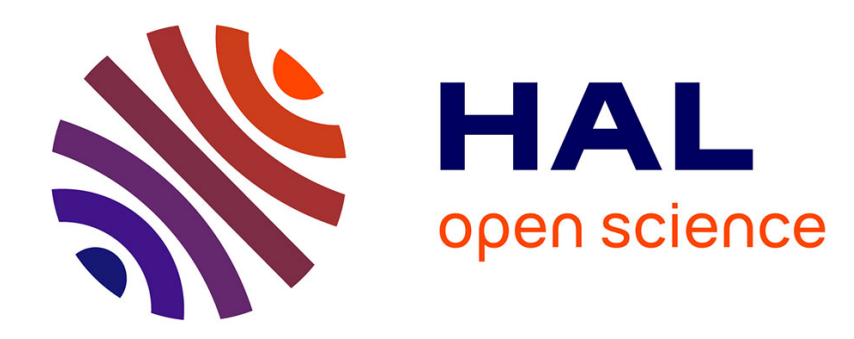

\title{
Systematics of fragment observables
}

B. Tamain

\section{- To cite this version:}

B. Tamain. Systematics of fragment observables. European Physical Journal A, 2006, 30, pp.71-79. 10.1140/epja/i2006-10108-7 . in2p3-00118530

\section{HAL Id: in2p3-00118530 https://hal.in2p3.fr/in2p3-00118530}

Submitted on 6 Dec 2006

HAL is a multi-disciplinary open access archive for the deposit and dissemination of scientific research documents, whether they are published or not. The documents may come from teaching and research institutions in France or abroad, or from public or private research centers.
L'archive ouverte pluridisciplinaire HAL, est destinée au dépôt et à la diffusion de documents scientifiques de niveau recherche, publiés ou non, émanant des établissements d'enseignement et de recherche français ou étrangers, des laboratoires publics ou privés. 


\title{
Systematics of fragment observables
}

\author{
B. Tamain ${ }^{\mathrm{a}}$
}

${ }^{a}$ LPC-ENSICaen, 14050 Caen cedex, France

Multifragmentation is observed in many reaction types: light ion induced reactions at large incident energies (in the $\mathrm{GeV}$ region), central heavy ion collisions from 30 to $100 \mathrm{MeV} / \mathrm{u}$, and peripheral heavy ion collisions between 30 and $1000 \mathrm{MeV} / \mathrm{u}$ or above. When nucleus-nucleus collisions are considered, another entrance channel parameter is the corresponding mass asymmetry. The first question which is addressed in this contribution is : do we observe similar reactions in each case? Multifragmentation may be related to a phase transition of nuclear matter. Some others features indicate that dynamical features are dominant. It is a priori possible that the underlying mechanisms are different in proton and nucleus induced reactions, in central and in peripheral collisions, at limited and at large bombarding energies. In order to see to what extent they can reflect similar behaviour, it is useful to compare the results of various reactions. The observables can be the fragment multiplicity, the mass distributions or the kinematical properties. In this contribution, we are looking for such general features. We will limit the discussion to the observations themselves, rather than the interpretation, which is the subject of numerous entries in this volume. The experimental results indicate that multifragmentation exhibits at the same time universal and entrance channel dependent properties.

\section{The necessity and the difficulty of the sorting}

A first difficulty in compring nucleus-nucleus collision data lies in the fact that they can differ significantly according to the impact parameter. Now, the impact parameter cannot be directly measured: it can be only estimated from other more direct observables. Depending on the experiment, various sorting parameters have been used: neutron or light charged particle (LCP) or total charged particle multiplicity[1-4], or LCP (or total) transverse energy[5], or flow angle[7], or specific quantities like Erat (ratio between the total perpendicular and parallel kinetic energy)[8] or $Z_{\text {bound }}$ (the total charge bound in fragments) $[9,10]$. One may also use more sophisticated methods as the principal component analysis method[11] or calorimetry[12,13] (see also chapter X in this volume).

The sorting aims either at following the evolution of the mechanism when the violence of the collision is increased (from peripheral to central collisions for nucleus-nucleus collisions), or at selecting something which is generally labelled "a source". An example is the selection of central collisions in nucleus-nucleus collisions. In the previous sentences, we have two concepts: "the collision violence" and "the source".

The violence is linked with the proportion of the initial aligned energy (the kinetic energy of the beam) that is shared among other degrees of freedom. It may be linked 
with a thermal energy if the available phase space is fully explored for the ensemble of selected events. A "source" is a piece of nuclear matter that is localized in momentum space. It is not necessarily equilibrated.

An important question is the quality of the sorting: to what extent is the selection efficient? The sorting cannot be precise for several reasons: finite size effects; detection inefficiency (dead areas and thresholds); fluctuations in the energy sharing in multi-source processes (for instance in binary processes). One may have an idea of this precision by looking at the correlation between various sorting variables. Data have been obtained for instance at MSU[1] in which particle multiplicities and transverse energies have been correlated. Another example has been obtained by the Indra-Aladin collaboration[14]: in this case, binary symmetrical collisions have been studied and transverse energy correlations have been obtained between the projectile-like (PLS) and the target-like (TLS) sources. The correlations are not better than about 20 percent. This means that sorting is efficient but not very precise. In any case, the detection has to be as complete as possible. It is possible to study the continuous evolution of the sorting variable keeping in mind that some mixing cannot be avoided. It is difficult to isolate a definite class of events without encountering one or another drawback: either a mixing with other event classes; or a cut in the available phase space for the selected event class. This difficulty is very well understood in simulations. A sorting from a mixing of various variables (principal component analysis) can slightly improve significantly the quality of the selection[11].

\section{Fragment observables.}

The raw multifragmentation observables are multiplicities, mass or charge distributions, isotopic distributions, kinetic energy and angular distributions. They can have various meanings depending on the collision nature: nucleus-nucleus collision versus light projectile (protons or pions, ...) induced reactions; peripheral versus central collisons.

Various observables can also magnify different collision features. This can be illustrated from what is well known at low bombarding energies, below $10 \mathrm{MeV} / \mathrm{u}$. In this case, deep inelastic reactions are dominant and it is well known that, depending on the observable, one is focussing on various aspects of the collision: fragment angular and kinetic energy distributions (Wilczinski plots) reflect the dynamics of the process: complete damping and isotropy is not observed for most of the events. On the other hand, mass transfer is described with Fokker Planck equations for which some degrees of freedom (the fast ones) are thermally treated (heat bath) whereas some others (mass transfer) are slowly evolving and do not reach necessarily equilibrium.

\section{Fragment production: a hierarchy.}

At low bombarding energy, it is well known that the decay of an excited nucleus ends with residue production. This decay product has a specific role among all the disentagration products. This feature is clear at low energy. A recent compilation[15] and the results of figures 1 and 2 indicate that this specific role of the largest fragment is often evidenced. It is the reason why, in the next sections, one will distinguish the largest fragment from the others. 


\begin{tabular}{|c|c|c|c|c|}
\hline System & method & $Z_{\text {sour }}$ & $E^{*} / u(M e V)$ & $\left(M_{I M F}-1\right) / Z_{\text {sour }}$ \\
\hline$\pi+\mathrm{Au} 8 \mathrm{GeV} / \mathrm{c}[12]$ & cal & 67 & 4 & 0.022 \\
\hline $\mathrm{Cl}+\mathrm{Au} 43 \mathrm{MeV} / \mathrm{u}$; periph; [17] & cal & 17 & 4 & 0.035 \\
\hline Ge+Ti35MeV/u; periph; [17] & cal & 32 & 4 & 0.035 \\
\hline Nb+Mg30MeV/u; central; [18] & cal & 45 & $3.4-3.8$ & 0.014 \\
\hline $\mathrm{Au}+\mathrm{Au} 35 \mathrm{MeV} / \mathrm{u}$; periph; [19] & cal & $\approx 75$ & 4 & 0.030 \\
\hline $\mathrm{Au}+\mathrm{Au} 600 \mathrm{MeV} / \mathrm{u}$; periph; [10] & cal & $\approx 75$ & 4 & 0,035 \\
\hline System & method & $Z_{\text {sour }}$ & $E^{*} / u(M e V)$ & $\left(M_{I M F}-1\right) / Z_{\text {sour }}$ \\
\hline$\pi+\mathrm{Au} 8 \mathrm{GeV} / \mathrm{c}[12]$ & cal & 59 & $7-8$ & 0.068 \\
\hline $\mathrm{Cl}+\mathrm{Au} 43 \mathrm{MeV} / \mathrm{u}$; periph; [17] & cal & 17 & 8 & 0.071 \\
\hline Ge+Ti35MeV/u; periph; [17] & cal & 32 & 8 & 0.071 \\
\hline $\mathrm{Ni}+\mathrm{Au} 90 \mathrm{MeV} / \mathrm{u}$; central; [11] & $\mathrm{cal} / \mathrm{SMM}$ & 86 & 7.5 & 0.070 \\
\hline $\mathrm{Xe}+\mathrm{Sn} 50 \mathrm{MeV} / \mathrm{u}$; central; [11] & $\mathrm{cal} / \mathrm{SMM}$ & 85 & $7-8$ & 0.074 \\
\hline Xe+Sn80MeV/u; periph; [20] & cal & 48 & 8 & 0.077 \\
\hline $\mathrm{Au}+\mathrm{Au} 80 \mathrm{MeV} / \mathrm{u} ;$ periph; [20] & cal & 70 & 7 & 0.069 \\
\hline $\mathrm{Au}+\mathrm{Au} 600 \mathrm{MeV} / \mathrm{u}$; periph; [10] & cal & 55 & 8 & 0.073 \\
\hline $\mathrm{Au}+\mathrm{C} 1000 \mathrm{MeV} / \mathrm{u}$; semi-periph; [4] & cal & $53-40$ & 7.5 & 0.10 \\
\hline La+C1000MeV/u; semi-periph; [4] & cal & $40-34$ & 7.5 & 0.077 \\
\hline Kr+C1000MeV/u; semi-periph; [4] & $\mathrm{cal}$ & $26-23$ & 7.5 & 0.07 \\
\hline
\end{tabular}

Table 1

This table is a non exhaustive compilation of many experiments in which the IMF multiplicities have been measured as a function of the excitation (dissipated) energy. The systems involved are indicated in the first column. The projectiles can be light (pions) or heavy (up to gold nuclei); the selected collisions can be central (one single source), or peripheral (projectile like source). The references are also indicated in the first column. The second column indicates the method that has been used to determine the excitation energy of the third column. Two excitation energies have been selected: around $4 \mathrm{MeV}$ and aroud $8 \mathrm{MeV} / u$, corresponding to close to and above the multifragmentation threshold. The fragment multiplicities (except for the heaviest fragment) are normalized to the source size in the last column.

\section{Fragment production: multiplicities.}

We label "fragments" as the detected products with a charge number of at least 3, which are generally named intermediate mass fragments (IMF). The lighter products (Z smaller than 3) are labelled light charged particles (LCP). The fragment multiplicity is $M_{I M F}$.

To what extent is $M_{I M F}$ correlated with energy dissipation? At low bombarding energies, it is established that $M_{I M F}-1$ is close to zero since no IMFs are emitted other than the residue: only LPCs remove the deposited energy. The situation is more complicated for larger energy deposition for which the preequilibrium energy contribution is significant and not uniquely defined. It has to be subtracted. After this subtraction, excitation energy is usually measured by calorimetry. It can be also obtained from the comparison with 


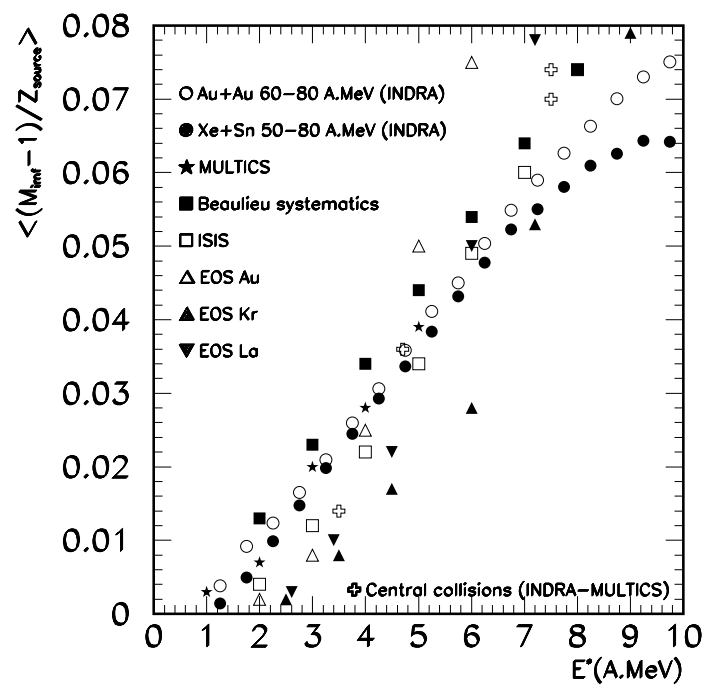

Figure 1. Correlation between $\left(M_{I M F}-\right.$ $1) / Z_{\text {sour }}$ (ordinate) and the dissipated energy (abscissa). The excitation (dissipated) energy has been corrected for preequilibrium and for expansion (if any: it is especially the case for the EOS data.) In that sense, the dissipated energy is mainly thermal. Several systems have been "summarized" in a single data set when the results were very close: it is the case for the Laval and Aladin data[12] and for the INDRA@GSI data[20].

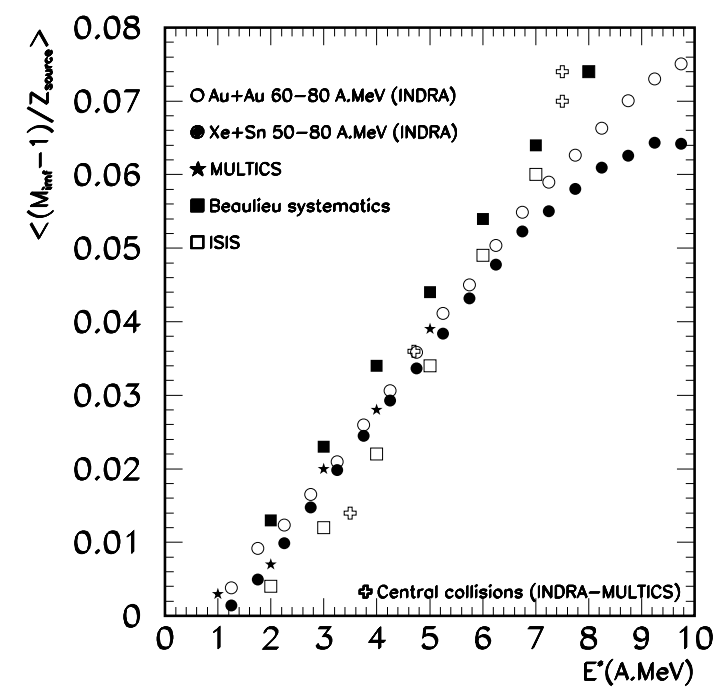

Figure 2. This figure is similar to the previous one but the EOS data have been removed. It turns out that the coherence between various reactions is very good in spite of the fact that one has included in the figure peripheral and central collisions, light and heavy-nucleus induced rections.

a model (for instance SMM[16]) in which equilibrium is assumed. When the bombarding energy is large, some compression effect may also be present and the corresponding expansion energy can be taken away. All these procedures can be disputed. Nevertheless, we have compared many data obtained in various ways to try to evidence some general behaviours. In table 1 , such a compilation is shown for two values of the "measured" excitation energy: $4 \mathrm{MeV} / \mathrm{u}$ and $8 \mathrm{MeV} / \mathrm{u}$. The list is not exhaustive. Since the expansion energy has been subtracted, the word "thermal" energy could be more appropriate but its use can be considered as too precise. For this reason, we will use the word "dissipated" for which the concensus may be better obtained. Very different reaction types are considered in table 1: pion induced reactions, central or peripheral heavy ion reactions, intermediate $(35 \mathrm{MeV} / \mathrm{u})$ or large $(1000 \mathrm{MeV} / \mathrm{u})$ bombarding energies. The method used to estimate the excitation energy can be calorimetry or comparison with SMM (third column). The source size $Z_{\text {sour }}$ is also estimated in various ways. Nevertheless, it appears that the ratio $\left(M_{I M F}-1\right) / Z_{\text {sour }}$ seems to be about the same for a defined excitation energy. This result is a first indication that multiframent production could be correlated with the dissipated energy. 
This tendancy is confirmed from figures 1 and 2 which show the correlation between $\left(M_{I M F}-1\right) / Z_{\text {sour }}$ and the measured excitation (dissipated) energy. All the systems considered in table 1 have been used. In order to clarify the figure, several systems are sometimes "summarized" by a single result. This is the case for the Indra@GSI data or for the Laval+Aladin data[17]. The general tendency is again the same for any system whatever the entrance channel is: light or heavy projectile; low or large incident energy; central or peripheral collisions (see also ref [21]). The coherence is especially good for high excitation (dissipation) energy. The results plotted in figures 1 and 2 indicate a continuous increase of the ordinate. One knows also that at larger dissipations, the fragment multiplicities decrease : i.e. the rise and fall of multifragment emission[9,4] for which a universal behaviour is also established.

Fragment multiplicities are hence correlated with the energy dissipated in the collision. This property has sometimes been described in terms of reducibility $[5,6]$ in the sense that the probability for emitting several fragments can be reduced to the probability for emitting a single fragment and to the corresponding energy cost. Such a result is quite coherent with the above discussion of figures 1 and 2 .

Thus it seems that multifragment production is to a large extent defined by the energy dissipated during the collision. Of course, the correlation obtained from the data cannot be perfect for two reasons. First of all, it is impossible to measure properly the "dissipated" energy because not possible to separate clearly in the data the relative contributions of preequilibrium, compression or thermal parts. A second feature is that many aspects of the collisions reflect an important role of the dynamics which is observed in mid-rapidity and in forward-backward emissions. These contributions are, to a large extent responsible for the deviations observed between the data at low dissipations in figures 1 and 2. They are discussed in the next section.

\section{Preequilibrium emissions.}

\subsection{General observations.}

Preequilibrium emissions correspond to particles or fragments that are not randomly emitted from identified sources (no isotropic emission in the plane perpendicular to the angular momentum). Besides the key quantities which are energy and angular momentum, they have kept some memory of the entrance channel, i.e. of the beam direction and/or velocity. From the time scale point of view, preequilibrium particles are emitted early. Their center of mass kinetic energies are generally larger than expected after full equilibrium reflecting the fact that the incident beam energy has not been shared among all the available degrees of freedom. The energy relaxation step bring energy in various degrees of freedom: the stored energy can be thermal if the whole available phase space has been occupied. The energy can also partially be stored as compression energy of nuclear matter, thus leading to an additional expansion contribution. A fraction of the available energy can also be stored as deformation energy of the hot source. The distinction between preequilibrium, expansion and thermal contributions is not trivial since the mean thermal decay time becomes very short for large excitations. 


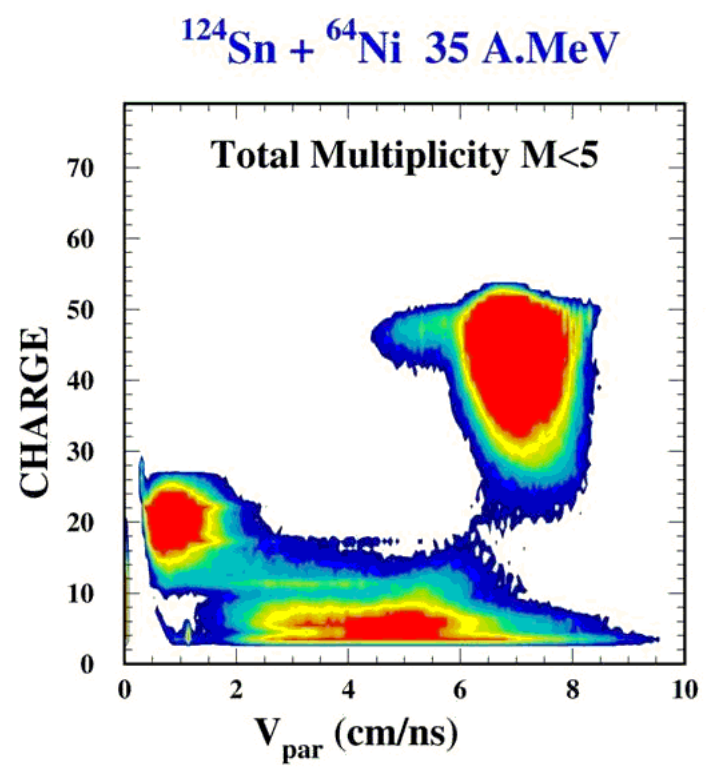

Figure 3. Correlation between the charges and the velocities of products emitted in semi-peripheral nucleusnucleus collisions. Mid rapidity is evidenced for light IMF. Similar results are published in reference[23].

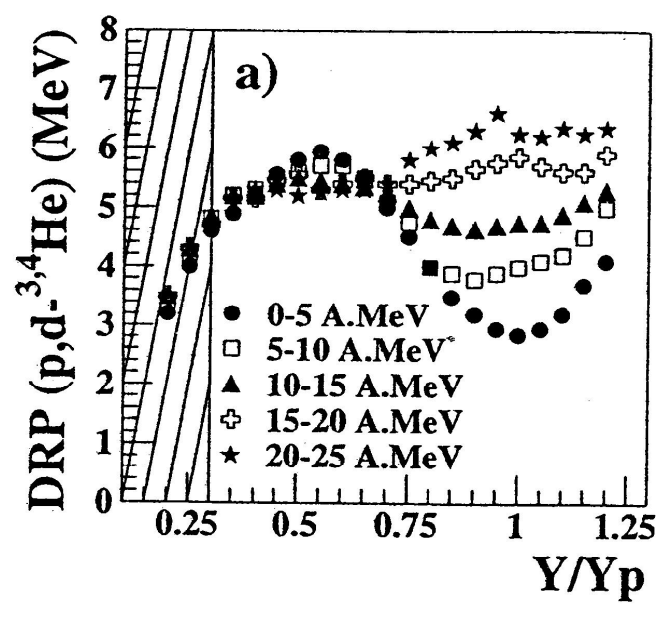

Figure 4. Abscissa: rapidity of selected LCP in beam rapidity unit; ordinate: double ratio parameter $(\mathrm{p}, \mathrm{d}, \mathrm{He}$ thermometer) corresponding to the abscissa rapidity. Various curves correspond to various energy dissipations (see ref.[26] for details). The dissipated energy has no influence on the results obtained at mid-rapidity.

\subsection{Angular distributions as signatures of preequilibrium.}

Two kinds of preequilibrium emissions are recognized in the data. In particle-nucleus or in central nucleus-nucleus collisions, preequilibrium LCP angular distributions are forward or backward peaked relative to the beam direction.

In semi-peripheral reactions, mid-rapidity (neck emission) occurs both for LCP and IMF. Preequilibrium LCP result mainly from direct nucleon-nucleon collisions in the overlap zone (see section 5.3). Concerning fragments, a general observation is that the largest decay fragment from a projectile-like source (PLS) is mostly faster than the lighter IMFs that are detected forward in the c.m. frame[22,23]: these lighter IMFs are accumulated close to the backward part of the Coulomb ring associated to the PLS whatever the bombarding energy is[24]. If the incident energy is limited $(40 \mathrm{MeV} / \mathrm{u}$ or below), this backward part of the coulomb ring is close to the c.m. velocity (mid-rapidity). The data of figure 3 correspond to this situation. Neck emission is clearly an entrance dynamical effect that leads to ambiguities in the measurement of the dissipated energy in a projectile-like source. It affects the projectile-like source velocity if it is reconstructed from the detected fragments. It affects also the excitation energy calculated from calorimetry. This ambiguity is larger when neck contribution is a sizeable fraction of the whole total yield. This is especially true for limited excitations and for symmetric heavy ion collisons. This can 
explain partially the relative dispersion of data in figures 1 and 2 at limited dissipations.

Depending on the observable, one may focus more or less on dynamical features. Neckemission is used in this context. On the contrary, one may subtract identified preequilibrium particles to try to isolate sources and try to get their excitation energies. Finally, one may select events for which the preequilibrium energy is small and can be neglected[2,30]. This procedure is never perfect especially for symmetric collisions in the entrance channel. Neverteless, it is possible to isolate events for which most of the available energy has been shared among many degrees of freedom. The deviations from full exploration of the available phase space can be to some extent "summarized" in collective variables such as deformation or expansion, which can be associated to lagrange parameters[25].

The fact that the results of figures 1 and 2 are coherent indicate that extracting dissipated energies from the data is a meaningful procedure. Similarly we will see in section 6 that the released IMF observables indicate that the process reflects to a large extent the available phase space.

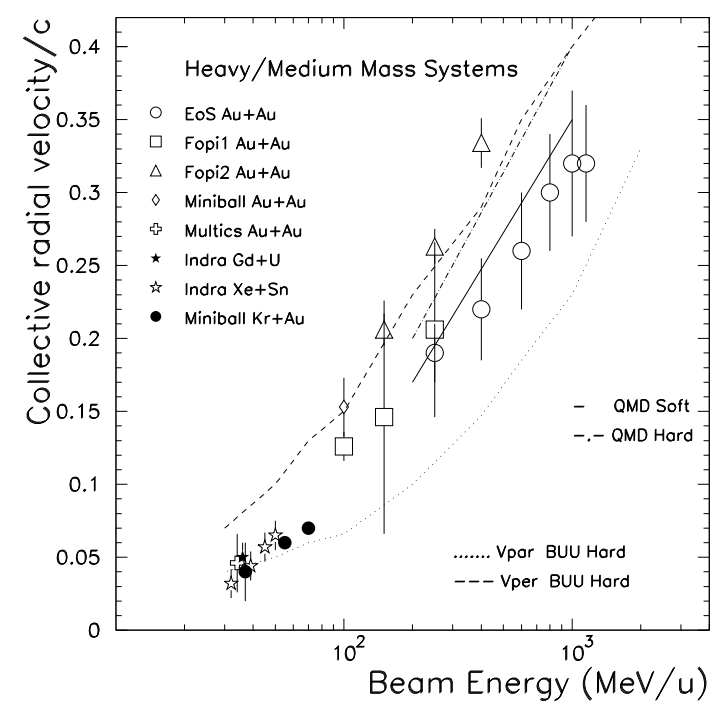

Figure 5. Non exhaustive compilation for the collective radial velocity as a function of the beam energy for medium- and heavy-mass systems in central collisions. Lines correspond to the predictions of transport models.

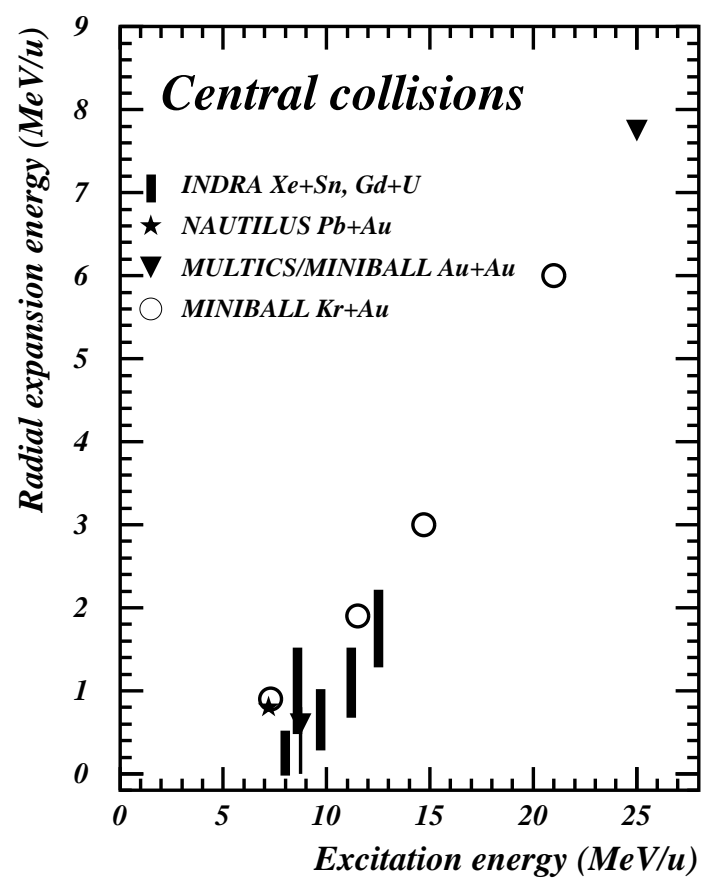

Figure 6. Systematics of the collective expansion energy as a function of the dissipated energy per nucleon in central collisions $[29]$.

\subsection{Kinetic energies as signatures of preequilibrium.}

Another indication of preequilibrium can be found in the measured kinetic energies of the emitted LCP and IMF. In semi-peripheral collisions, entrance channel effects are 
clearly evidenced[26,27]. For instance, in ref.[26], it is shown that the transverse LCP energy at mid-rapidity does not depend on the violence of the collision at variance with the energy of LCP emitted from the PLS (figure 4). LCPs emitted at mid-rapidity reflect the incident energy per nucleon and the Fermi motion of the projectile and target nucleons whereas LCPs emitted at velocity closer to the PLS one reflect the dissipated energy. Depending on the location in the velocity plane, we observe entrance channel or dissipation effects.

In central collisions, the LCP kinetic energy spectra exhibit non-maxwellian shapes especially along the beam direction. Many data have been interpreted in unfolding the measured spectra in order to separate two components: preequilibrium on the one side and an equilibrated part on the other side. Their relative contributions depend strongly on the emission angle which is a help to succeed in the unfolding. For the equilibrated part, the mean c.m. kinetic energy $\langle\epsilon>$ depends on the mass of emitted LCP or IMF. This result indicates that a non thermal component is present. It is generally attributed to an expansion energy reflecting nuclear matter compression properties. Figure 5 is a non exhaustive compilation showing that expansion energy (or radial velocity) is small for incident energies lower than $30 \mathrm{MeV} / \mathrm{u}[28]$. Conversely, figure 6 indicates that expansion is significant for measured excitation (deposited) energies exceeding 5 to $6 \mathrm{MeV} / \mathrm{u}[29]$.

\section{Charge or mass distributions.}

We have already noticed in section 3 that the heaviest fragment emitted from a selected source plays a significant role among all the outgoing fragments. This is the case at excitation energies below the multifragmentation threshold since, in this case, the largest fragment is an evaporation residue. When multifragmentation occurs, the largest fragment has no longer this specific role and its mass becomes much lower. It is observed in the experiments that this change from evaporation-like events to multifragmentation is rather abrupt when the dissipation is increased. In some cases, the coexistence of evaporationlike and multifragmentation events has been observed for comparable dissipations: it is the bimodality signal that is a possible signature of a phase transition of the system (see O. Lopez and MF Rivet, this volume). It is only stressed here that bimodality can be a first indication of a statistical behaviour for defining the masses or charges of the products released in nucleus-nucleus collisions.

More generally speaking, many data indicate that the overall charge and mass distributions can be described by statistical models, i.e. in models in which the main ingredient is the available phase space. This is true for the total charge distribution $[2,30]$ and for the distributions associated with the largest or the second and third largest fragment [31,32]. This is true for limited excitations[2] for which few fragments are released up to very large ones leading to vaporization[33]. In this last case, only LCP are detected but their relative abundances are also understood in a statistical approach[34]. An interesting compilation is shown in figure 7. It concerns several experiments with quite different entrance channels and for which the measured mass distributions seem to reflect mainly the deposited energy in $\mathrm{MeV} / \mathrm{u}$. Similarly, it has been shown in ref.[2] that similar results are found in peripheral and central collisions, indicating that the dissipated energy seems again to be the main ingredient which defines the splitting of the system. 

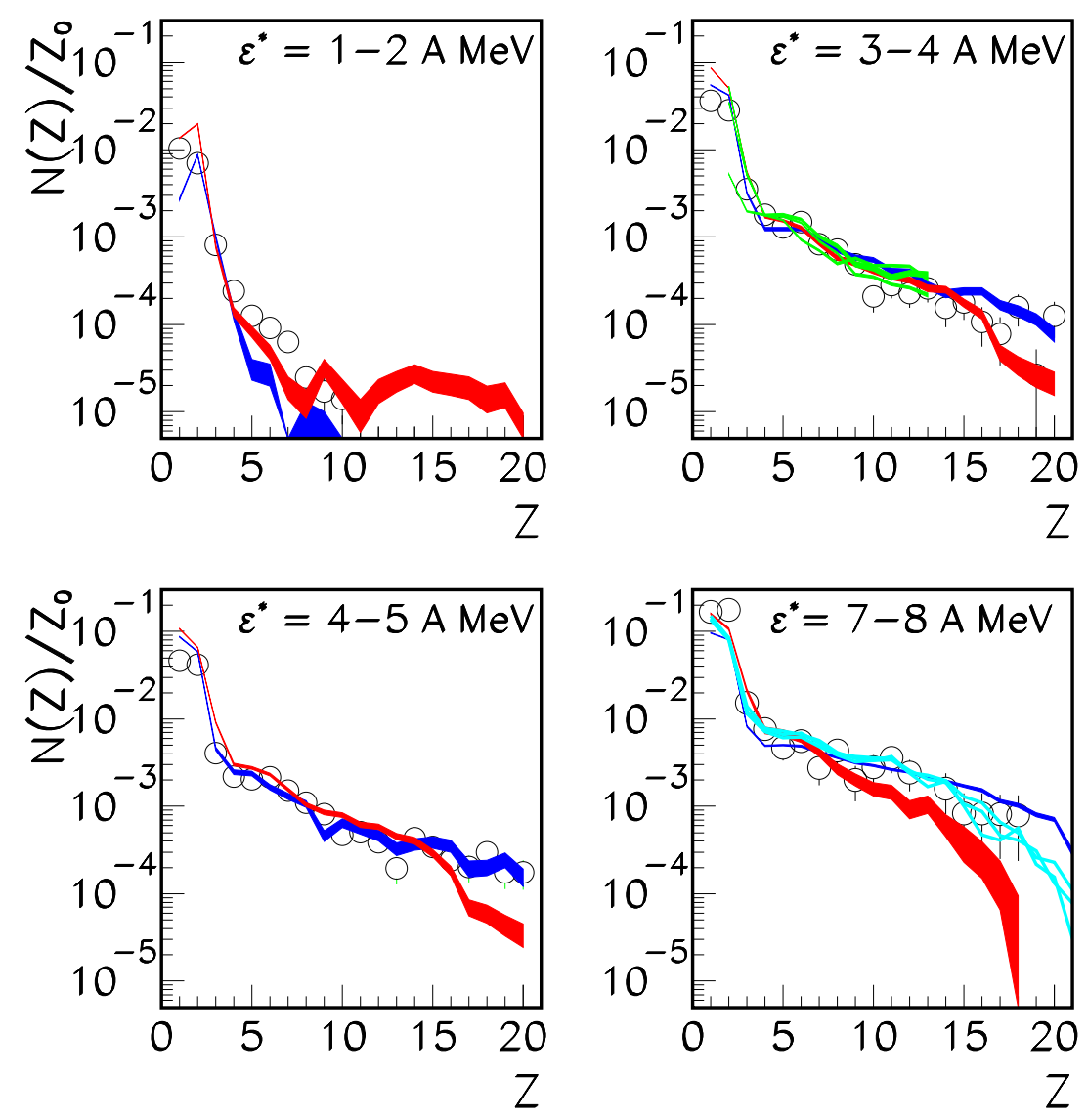

Figure 7. Mass distribution for various excitation energy ranges obtained in several experiments involving very different entrance channels. Dark blue curve: $\mathrm{Au}+\mathrm{Au}$ central collisions from multics data; green curve: Fasa data; light blue: $32 \mathrm{MeV} / \mathrm{u} \mathrm{Xe}+\mathrm{Sn}$ Indra data; red: ISIS data. This figure has been prepared by M. Dagostino[35].

Many results are reproduced by models like SMM[16] or MMMC[36] in which full statistical equilibrium is assumed. Of course, one may argue that such agreements can be obtained only by adjusting parameters such as the density at freeze-out. The total mass and the excitation energy of the initial source are also adjusted to reproduce the data, but their values are in agreement with calorimetric measurements when they are available. The excitation energies and source masses are smaller than the available energy and mass simply because of preequilibrium emission. More direct data are also available in which several systems are compared independently of a model. For instance, in ref. [37] it is shown that the systems $\mathrm{Xe}+\mathrm{Sn}$ and $\mathrm{Gd}+\mathrm{U}$ exhibit similar mass distributions at similar measured excitation energies in $\mathrm{MeV} /$ nucleon. Similarly, in ref.[11], central $\mathrm{Xe}+\mathrm{Sn}$ and $\mathrm{Ni}+\mathrm{Au}$ collisions (same fusion-like source mass at similar excitation energies) exhibit similar mass distributions. This dominance of phase space is also evidenced by the fact that the observed multifragmentation mass distributions can be reproduced simply in 


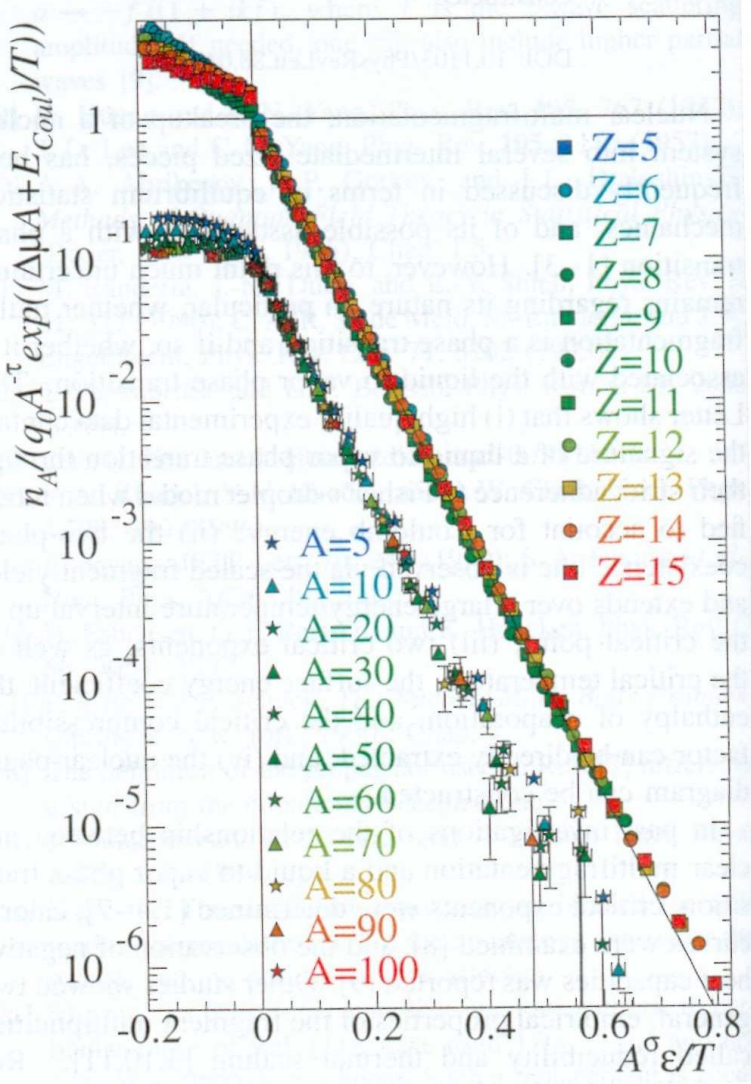

Figure 8. Analysis of the ISIS data showing that the probability for emitting a given fragment can be fitted in the Fisher formalism in which the emission is mainly governed by statistical properties of nuclei[39].

cutting at random a rope in a number of elements equal to the observed multiplicity[38]. The multifragmentation mass distribution would hence be constrained only by the mass conservation for a given fragment multiplicity.

One of the most spectacular results indicating a statistical behaviour is the reducibility property[6] which indicates that fragment production probabilities can be put together in Arrhenius plots and the very beautiful fits obtained in the so-called Fisher scaling analysis. Figure 8 is the most famous one but similar fits have been obtained with other data[40]. Even if such an analysis relies on several adjusted parameters (which are consistent with theoretical expectations) and in spite of the blurring effects of secondary decay, this property is further evidence of statistical behaviour.

An isospin analysis of the released products is also in agreement with this statement[41, 42]: isoscaling is the observation that the probability ratio for producing a defined isotope in two different reactions may be expressed as:

$R_{1,2}=\exp (\alpha N+\beta Z)$

where $N$ and $Z$ are the numbers of neutron and proton of the isotope. Even if the physics is not transparent for the values of the parameters $\alpha$ and $\beta[43]$, the validity of equation 


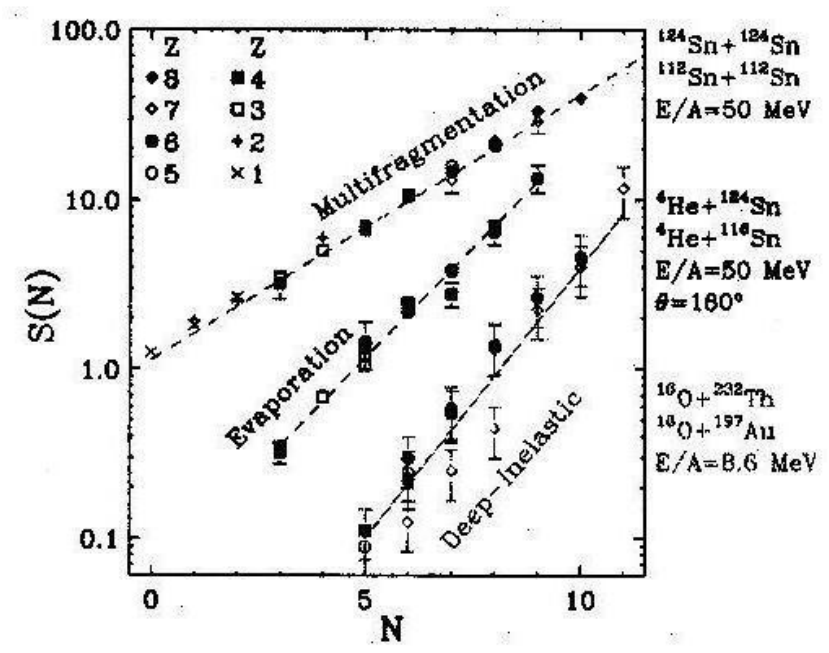

Figure 9. Production ratio for various isotopes and several reaction pairs. The isoscaling as a function of the neutron number is observed for very different reaction types, from evaporation and deep inelastic collisions to multifragmentation. See text for details. Extracted from ref. [41].

1 indicates that statistical features are present everywhere. Figure 9 is an illustration showing that this description is valid over a wide range of incident energies and reactions. In this figure, $R_{1,2}$ has been multiplied by $\exp (\beta Z)$ in order to express the results only as a function of $N$. Scaling is observed for deep inelastic collisions, for evaporation and for multifragmentation as well. It is coherent with the fact that all these processes are governed by the available final states[45]. It does not seem that the sequential decay affects significantly the results[44]. Nevertheless, such observations do not mean that full equilibrium is achieved. On the contrary, some FOPI data[8] indicate that the full mixing between the projectile and the target is not achieved even in the most central collisions. Hence the available phase space is widely opened but is still constrained by some entrance channel memory.

\section{Conclusion}

From many data, fragment production exhibits both dynamical and statistical aspects. The multiplicity is mainly governed by the dissipated energy. It increases from a single residue (or two fission fragments) for limited excitations up to large values in the multifragmentation regime, the rise and fall leading to a vanishing multiplicity when the dissipated energy is sufficient to allow vaporization. In the multifragmentation case, some fragments can be released in dynamical processes such as neck emission observed in semiperipheral collisions. In any case, fragments are accompagnied by multiple light particles, some of which show dynamical features.

The size distributions of the detected fragments are also mainly governed by the available phase space; the heaviest fragment has specific properties at least for limited excitations, below 3-5 MeV/u, i.e. below the threshold energy for which the multifragmentation 
channel sets in significantly. Above this threshold, the specificity of the heaviest fragment is weaker.

However, many kinematical properties of the fragments reflect dynamics in the sense that they have retained some memory of the entrance channel. This is clearly the case for their angular distributions and also for their kinetic energies which are not purely thermal for nucleus-nucleus collisions at bombarding energies exceeding $50 \mathrm{MeV} / \mathrm{u}$ even if central collisions are selected. This deviation from a thermal behaviour can sometimes be interpreted as a collective deformation[46] or compression effect initiated by the early compression phase in the collision. In such a case, a statistical description can be used provided that one introduces in the description a constraint summarizing the dynamical behaviour.

\section{REFERENCES}

1. L. Phair, et al., Nucl. Phys. A548 (1992) 489.

2. M. d'Agostino, et al., Nucl. Phys. A724 (2003) 455.

3. R. Wada, et al., Phys. Rev. C69 (2004) 044610.

4. L. Hauger et al. (EOS coll.), Phys. Rev. C62 (2000) 024616.

5. L. Moretto, et al., Phys. Rev. Lett. 74 (1995) 1530.

6. L. Moretto et al., Phys. Rep. 287 (1997) 249.

7. J. Frankland, et al. (INDRA coll.), Nucl. Phys. A689 (2001) 905.

8. F. Rami, et al. (FOPI coll.), Phys. Rev. Lett. 84 (2000) 1120.

9. P. Kreutz, et al. (ALADIN coll), Nucl. Phys. A556 (1993) 672.

10. W. Trautmann, et al. (ALADIN coll), Proceedings of the XXXIII Intern. Wint. Meet. on Nucl. Phys., Bormio, 1995, p.372.

11. N. Bellaize, et al. (INDRA coll.), Nucl. Phys. A709 (2002) 367.

12. L. Beaulieu, et al., Phys. Rev. C64 (2001) 064604.

13. Y.G. Ma, et al., Phys. Rev. C69 (2004) 031604.

14. M. Pichon, et al., unpublished data.

15. R. Bougault, et al., to be published.

16. J. Bondorf, et al., Phys. Rep. 257 (1995) 133.

17. L. Beaulieu, et al., PRC54(1996)R973.

18. L. Manduci, PhD, Caen, 2005.

19. M. d'Agostino, et al. (MULTICS coll.), Nucl. Phys. A724 (2003) 455.

20. INDRA-ALADIN coll.: unpublished results.

21. V. Metivier, et al. (INDRA coll.), Nucl. Phys. A672 (2000) 357.

22. J. Colin, et al., (INDRA coll.), Physical Review C67 (2003) 064603.

23. A. Pagano, et al., Nucl. Phys. A734, (2004) 504.

24. J. Lukasik, et al. (INDRA-ALADIN coll.), Phys. Lett. B566 (2003) 76.

25. P. Chomaz et al., Ann. Phys. 320 (2005) 135.

26. T. Lefort et al., Nucl. Phys. A662 (2000) 397.

27. J. Lukasik, et al. (INDRA-ALADIN coll.), Phys. Rev. C66 (2002) 064606.

28. D. Durand, Nucl. Phys. A 654 (1999) 273c, and D. Durand et al, Nuclear dynamics in the nucleonic regime, IOP Publishing Ltd, 2001.

29. M.F. Rivet et al, Proc. XXVII Winter Hirshegg meeting, ed. H. Feldmeier (1999). 
30. N. Marie, et al. (INDRA coll.), Phys. Lett. B391 (1997) 15, and Phys. Rev. C58 (1998) 256-269.

31. E. Geraci, et al. (CHIMERA coll.), Nucl. Phys. (2004) 524.

32. M. d'Agostino, et al., Nucl. Phys. A650 (1999) 329.

33. M.F. Rivet, et al., (INDRA coll.), Phys. Lett. B388 (1996) 219.

34. B. Borderie, et al., (INDRA coll.), Eur. Phys. Jour. 6 (1999) 197.

35. M. D'Agostino: unpublished.

36. D.H.E. Gross, Rep. Prog. Phys. 53 (1990) 605.

37. M.F. Rivet, et al. (INDRA coll.), Phys. Lett. B430 (1998) 217 and J. Frankland et al. (INDRA coll.), Nucl. Phys. A689 (2001) 940.

38. R. Dayras, unpublished calculations

39. J.B. Elliott et al., Phys. Rev. Lett. 88 (2002) 042701.

40. N. Leneindre (INDRA coll.), to be published.

41. B. Tsang, et al., Phys. Rev. Lett. 86 (2001) 5023.

42. G.A. Souliotis, et al., Phys; rev. C68 (2003) 024605.

43. S.R. Souza, et al., Phys. rev. C69 (2004) 031607R.

44. M.B. Tsang et al, Phys. Rev. C64 (2001) 054615.

45. A.S. Botvina, et al., submitted to Phys. Rev. C.

46. A. Lefevre, et al. (INDRA-ALADIN coll.), Nucl. Phys. A735 (2004) 219. 